\title{
REFILATER-3D PRINTING FILAMENT EXTRUDER
}

\author{
ASTLE JOHN, HARIKRISHNAN V P, NAYANANETTO \& DR. G R GNANA KING \\ Sahrdaya College of Engineering and Technology
}

\begin{abstract}
Reuse and disposal of $3 D$ printing and prototyping waste is a major issue to all users of the device and designers alike, hence it is necessary to treat the generated plastic waste. Here we propose a solution to this recycling issue with a product that can recycle and output new filament from the existing waste filaments and plastic wastes of selected materials. The device can also produce new filament from the proper type of materials which ensures the recycling of plastic waste. The machine is a major link of closed loop recycling where the plastic used to make the filament is converted back to filament hence not producing or requiring extra plastic. The project also aims to produce recycled $3 D$ filament which can be sold commercially as a product to, designers and makers and all creative minds at a relatively less cost. This is done by firstly sorting the plastic into its types like PET, HDPE etc, next the plastic is color sorted and then shredded to thin particles, and is transferred to the heating and extruding block which extrudes the plastic without causing anyfumes.

The main innovative part of the product is its ability to spool the extruded filament automatically without human aid. This means that the filament is fed automatically to the

spooler and is spooled by the mechanism that automatically adjusts the tension of the filament and spools it and cuts off the spooler when no more filament comes out of the extruder. Hence the recycled filament is a takeaway product which can directly be used as a raw material for the printer. Here We see that the waste filament and selected materials of plastic is converted to useful filament in one continuous process which itself is the aim of the project.

KEYWORDS: Shredder, HDPA, Extruder, Hopper, Spooler, Guider
\end{abstract}

Received: Jun 08, 2020; Accepted: Jun 28, 2020; Published: Sep 01, 2020; Paper Id.: IJMPERDJUN20201006

\section{INTRODUCTION}

Finding ways to work with plastic waste is necessary. The entire ecosystems and societies are being threatened by the environment. It results in deterioration of our planet and life of the people. A material made to last hundreds of years, a fraction of its possible lifespan, used for only few minutes and these are quickly discarded. All the plastic seen to be lying around us are not waste, but just resources. These resources can become the beginning of something new, if they are treated properly. The planet as well as the society around us, is large. The resource lying around us everywhere can be made to become an educational tool or a source of income for our community.

Plastics as we know are synthetic chemicals that are generally extracted from petroleum and made of hydrocarbons (chains of hydrogen and carbon atoms). Plastics are normally long molecules, called polymer which is made of many repetitions of a simple molecule called a monomer. This is why the structure becomes mostly durable and long lasting.[1]Other advantages of plastic are their low cost, ease of manufacture and also versatility. This is the reason why they are used in an enormous and expanding range of products, from shampoos to space rockets. Since plastics are found everywhere, they are causing serious environmental concerns regarding its slow decomposition. Another reason for this is its strong bonding molecules. Depending on application as well as type of 
plastic, most plastics contain other organic or inorganic compounds blended with them called as additives to improve performance.

It is crucial to know what type of plastic we are dealing with. With this we can get to know the melting temperature in order to set exact temperature of the machine so that we can run a smooth recycling process. The different plastic types include PET (polyethylene terephthalate), HDPE (high-density polyethylene), PVC (polyvinyl chloride), LDPE (low-density polyethylene), PP (polypropylene), PS (polystyrene) and ABS, Acrylic or Polycarbonate are included in this category.

\section{LITERATURERE VIEW}

Plastic use and disposal is a major issue that still needs to be solved on the perspective of a more sustainable development of human activities. Circular economy has been proposed as a potential solution by using materials and energy through multiple phases in a recycling flow. [3]. However, most of the studies present in the literature of plastic recycling and other wastes are mainly focused on large centralized networks that try to efficiently carry their costs and / or income. In addition, particularly the centralized plastic recycling networks should face the challenge of collection and transportation for high volume and low weight polymers, affecting its economic feasibility. The emergence and development of Open-source technologies such as 3D printing extrusion and other devices provide an opportunity to explore distributed recycling as an alternative option [4]. Under this distributed model, users will collect domestic plastic waste themselves, which can be recycled at their nearest recycling points, in order to produce 3D plastic filament and thus contribute to the Circular Economy by reducing the plastic waste. Providing printable material to the printer whenever required is an important part of 3D printing. Normally inclusion of plastic filament reels is done, among which the most common is PLA. However, this is a final product that is marketed separately. Since this is a cost included method we find an alternative solution. That is in order to obtain printable material, the plastic material from disposable bottles are recycled and reused. Accordingly our present work shows the design of a 3D printer extruder with equipment to supply the filament for printing from different kinds of plastics. To integrate the supplying system of plastic material to the printer by supplying recycled plastic bottles which has to go through a whole process, and thus make the printer work as a single unit is the main objective.[5] The project also raises awareness in the filed of recycling materials mailny plastic to avoid pollution, and along with this, the famous three ecological R's that is Reduce, Reuse and Recycle can be put into practice.

The main advantage of our project is that it provides a closed loop recycling system that stresses upon zero waste concepts in our society promoting eradication of plastics around us.

\section{OVERVIEW}

The project REFILATOR aims to convert the waste plastic and failed 3D prints to usable printing filament. It mainly consists of three primary parts: shredder, extruder and the spooler. Firsly the waste filaments are collected washed and dried then the material is placed inside the shredder of the machine and the machine is turned on. The plastic is shredded to required proportions and is fed through an extruder. The extruder is the part which collects the waste shreads and feeds them to the heating area for it to be extruded out as filament. The heating and speed of extrusion is automatically controlled to ensure maximum efficiency. 


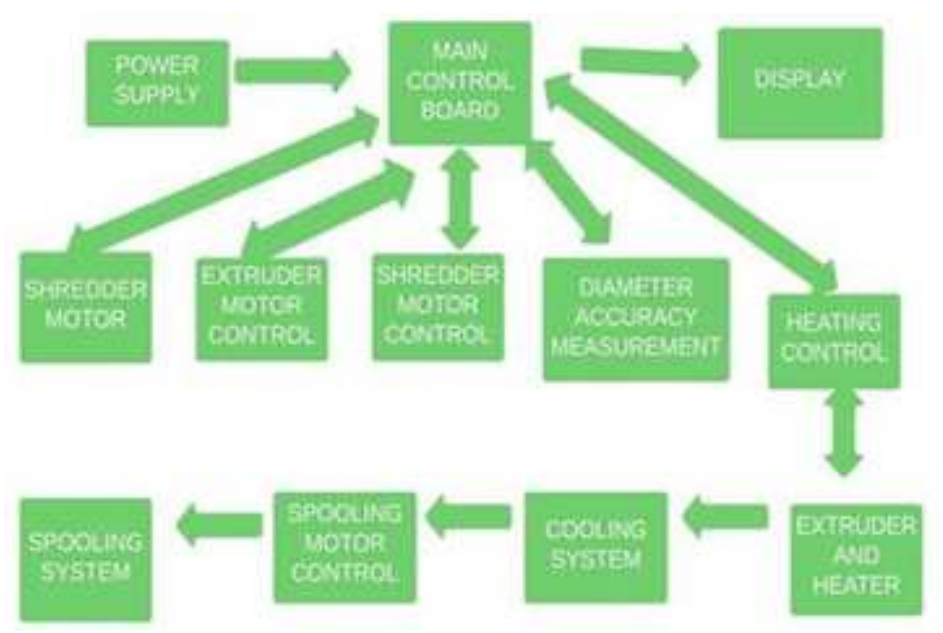

Figure 1: Block Diagram.

\section{DESIGN OF THE SYSTEM}

\section{Shredder}

The shredding system shreads the plastic into workable sizes. Plastic is put into the shredder so that it is grinded into small pieces, by the grinder. It also prevents large parts from reaching the extruder. It is then passed onto the extruder part.

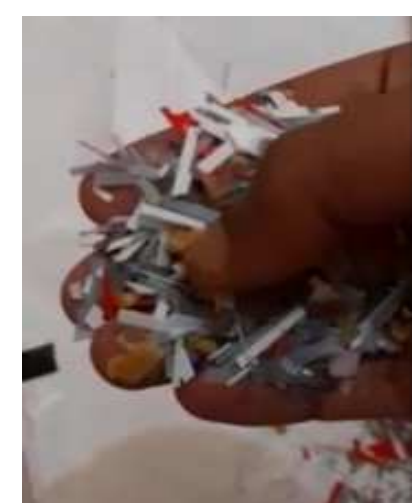

Figure 2: Shredder Output.

\section{Pelletizer}

This small part of the system is used to convert the left over parts to pellets for easy extrusion. This will also make the shredding process more precise and perfect, by cutting down them into more suitable small size.

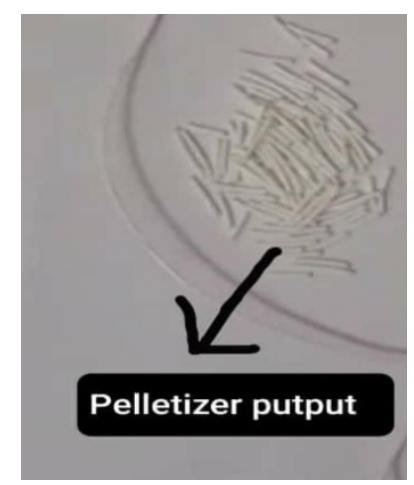

Figure 3: Pelletizer Output. 


\section{Extruder}

The small plastic pieces are heated into filament form, inside a barrel. The PID temperature controlled heating element provides even heating of the shredded plastic waste. It is made up of stainless steel, rust and corrosion proof anti-clogging. The design is done in such a way that it prevents jamming of filaments by sensing filament jamming and emergency stopping of the extruder motor. The extruded soft filament plastic is cooled down with the help of the cooling system and is drawn with the help of the guide rail system containing rollers to roll the filament towards the spooler. The speed of the rollers can be adjusted to control the thickness of the extruded filament.

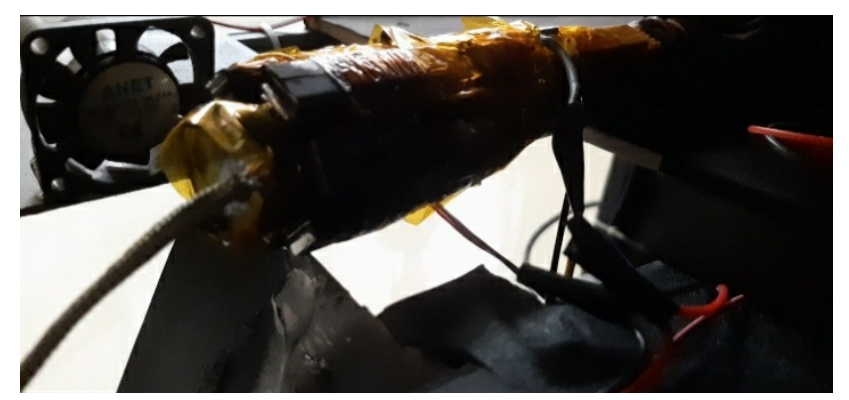

Figure 4: Extruder.

\section{Roller}

The main task of the roller is to guide the extruder filament from the extruder nozzle to the spooling station. This is done by rolling and guiding mechanism.

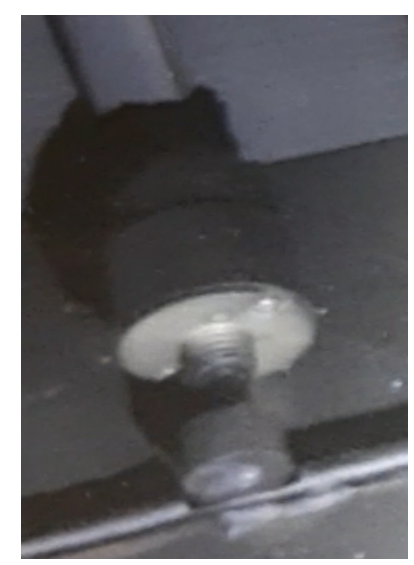

Figure 5: Roller.

\section{Optical Sensing Systems}

This system is used to automatically sense the filament and alerts the spooler to be switched on. Another advantage is that it senses the spooling rotations and stops when the spool is full. It will also automatically stops if no filament is sensed from the optical detector. The system stops after sensing the amount of filament in the spool. 


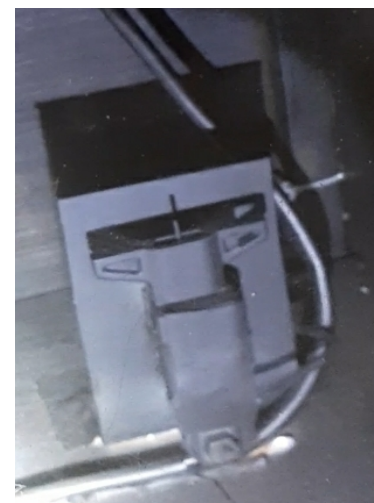

Figure 6: Optical Diameter Sensor.

\section{Spooler with Auto-Feeding Filament System}

The main highlight of the machine is the automation in spooler part. What it does is that it grabs the filament automatically after being signaled by the optical sensors. A director directs the filament onto the spool and the filament caught by the spooler is spooled. The spooling cuts of automatically as soon as the filament spool is almost full.

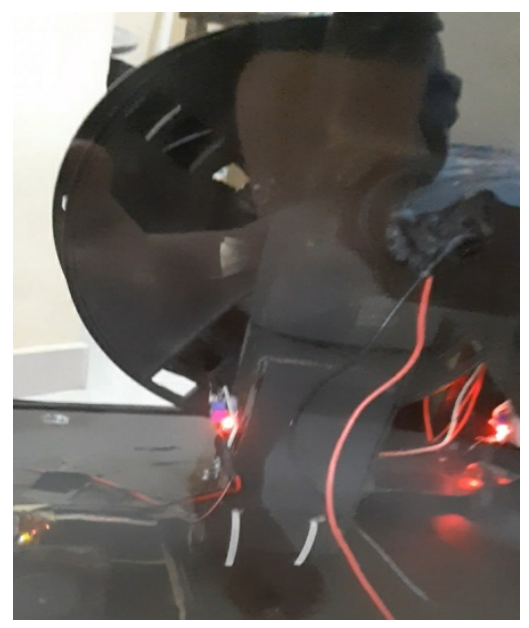

Figure 7: Spooling System.

\section{RESULTS AND DISCUSSIONS}

This project establishes an advantage and particularity unlike other 3D printing systems. The main idea behind this extruder system is to make recycling of disposable materials possible. In this work of ours, a literature review was conducted based on networks including plastic recycling. From this a conclusion, was made that no optimization model devoted to local and distributed recycling network existed for small- scale plastic waste. A suggestion is also made, that is research based on more plastic recycling stages rather than just collection and the simultaneous of more than one type of plastic. The project stresses on the zero waste concept, which the society looks forward to. Along with that by using a closed loop recycling system to generate $3 \mathrm{~d}$ printing filament from either plastic waste or used filaments, there is an assurance that the project will be a lot helpful for users to get $3 \mathrm{~d}$ printing filaments at cheap cost rather than the ones available in the market. 


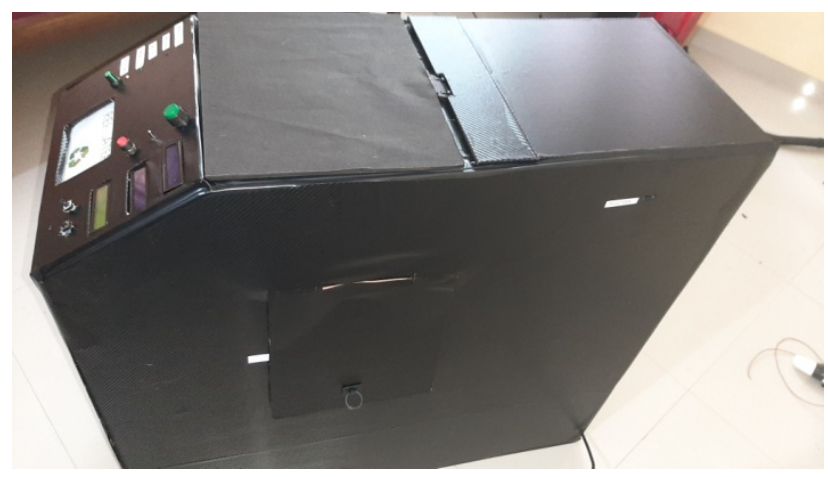

Figure 8: Refilater-Final Machine.

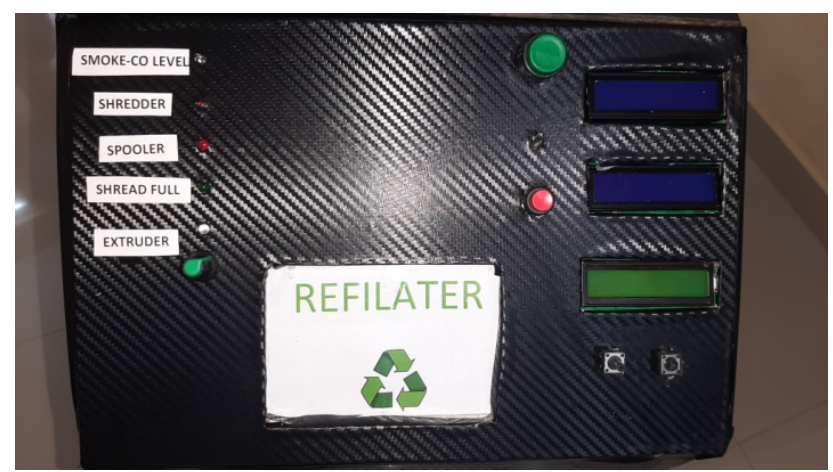

Figure 9: Refilater-Front Panel of the Machine.

\section{CONCLUSIONS}

In this project we have made the spooler that spools the extruded 3D printed filament. Automatic spooling is one of the main innovations in this project. It helps and makes the recycling and extrusion of the filament easier. The diameter monitoring system was developed using the help of a digital vernier caliper with $i 2 \mathrm{c}$ outputs. The data and clock outputs were used to measure the diameter using arduino software and the diameter was displayed real time on the LCD display. The threshold for the diameter thickness of the 3D printing filament was decided to be $1.69 \mathrm{~mm}$, with an idea thickness of $1.75 \mathrm{~mm}$. The material for the 3D printing filament recycler was decided to be HDPE plastics and 3D printing filament waste materials. The filament spooler was decided to be of cardboard material with a special valve, that accepts the filament only in one direction, hence assuring the proper attachment of the filament to the spooler. This ensures the proper spooling of the recycled 3D printing filament.

\section{ACKNOWLEDMENT}

We acknowledge with great gratitude all the faculties of Sahrdaya College of Engineering and Technology for giving us constant support and inspirations throughout the completion of the project.

\section{REFERENCES}

1. Ellen MacArthur Foundation, "Towards the Circular Economy," Ellen MacArthur Found., vol. 1, pp. 1-96, 2013.

2. M. Saidani, B. Yannou, Y. Leroy, and F. Cluzel, "How to Assess Product Performance in the Circular Economy Proposed Requirements for the Design of a Circularity Measurement Framework,"2017.

3. C.M.Angioletti,F.Sisca,R.Luglietti,and 
4. M. Taisch, "Additive Manufacturing as an opportunity for supporting sustainability through implementation of circular economies,” 2016 IEEE 2nd Int. Forum Res. Technol. Soc. Ind. Leveraging a better tomorrow, no. September, 2016.

5. N. Singh, D. Hui, R. Singh, I. P. S. Ahuja, L. Feo, and F. Fraternali, "Recycling of plastic solid waste: A state of art review and future applications,” Compos. Part B Eng., vol. 115, pp. 409-422, 2017.

6. Sridhar, N., \&Surendrakumar, A. (2017).Performance Evaluation and Modification of Shredder Cutting Mechanism. International Journal of Agricultural Science and Research, 7(5).

7. Bhattacharya, S. S., \&Chaudhari, S. B. (2015). Study on structural and thermal properties of polypropylene silica nanocomposite filaments. International Journal of Textile and Fashion Technology, 5(1), 15-22.

8. Freitas, I. C., \& Auil, F. (2013). Effect of Fixing Stage from Texturing Synthetic Filaments Yarn Process by False Twist Technology on the Polyester Yarn Skein Shrinkage. Technology (IJTFT), 3(2), 39-46. 

